\title{
The Present Situation and Management Measures of Adverse Drug Reactions in China
}

\author{
Jinguo Wang \\ Department of Urology \\ The First Hospital of Jilin University \\ Changchun, China \\ wangjinguolily@163.com
}

\author{
Na Wang* (corresponding author) \\ Department of Anesthesiology \\ The First Hospital of Jilin University \\ Changchun, China \\ wangna080613@163.com
}

\begin{abstract}
Adverse drug reaction monitoring work in our country has made many achievements, but there are still some problems in practice. With the international implementation of the adverse drug reaction monitoring earlier and more successful than countries, our country's adverse drug reaction monitoring work still exists some shortcomings and adverse drug reaction monitoring work faces many challenges. Establishment of a perfect reporting system, clear responsibility and liability are key components of effective management of adverse drug reaction.
\end{abstract}

Keywords—management; adverse reactions; government; drug

\section{INTRODUCTION}

According to medical practice, medical personnel have the duty to inform patients the possibility of adverse reactions. At present, our country's medical staff to undertake the main task of the adverse drug reactions report, report the number reaches more than $90 \%$ of the total amount of report. Thus medical staff overall cognitive water of adverse drug reactions will directly affect the quality of the whole medical treatment activity. So hospital to medical attention and support to the prediction of adverse drug reactions, and the adverse drug reaction monitoring work in department of medical quality is the most comprehensive target management appraisal system, establish and improve the rules and regulations and the hidden. Practical and will monitor the work carries out to use. Only the medical staff and improve the overall level of the cognition of adverse drug reactions, to fully improve the efficiency of diagnosis and treatment.

\section{ADVERSE DRUG REACTIONS}

Adverse drug reactions refer to the qualified drugs under normal usage and dosage has nothing to do with the purpose of or unexpected harmful response. In recent years, the frequent occurrence of adverse drug events in our country, each year more than 5000 person-times of hospitalized patients, which are associated with adverse drug reactions of patients, can reach more than 250, about 240000 patients died. A drug is approved, although before listing through rigorous clinical trials, but because of the limitations on experiment environment, object, when the drugs listed and the public in a big range after use, still may produce all sorts of unforeseen adverse reactions, and even show serious harmfulness [1]. Therefore, which requires the adverse drug reaction monitoring departments should increase of post-marketing drug carefully monitored, only use the scientific analysis methods to analyze clinical information acquired from evaluation, determine the internal causality, the objective of drug safety evaluation, and inform the doctors and the public, the related information to achieve the purpose of guiding the rational use of drugs, avoid adverse event to repeat.

With the wide use of drugs and in-depth study, people gradually found that the drug has the duality, namely in the oil that has remedial effect at the same time, there are a lot of adverse reactions. In 1961 after the occurrence of adverse drug events, people gradually realize the adverse drug reactions of harm. In modern medical development today, people are constantly plagued by adverse drug reactions. A large population in our country, the problem of adverse drug reactions is also increasingly prominent and severe. According to reports in the literature, our country each year because of drug adverse drug reactions and hospitalization of patients more than 5 million people, of which about 190000 people died. The deaf-mute children in 1990 , our country has more than 1.8 million people, drugs cause deaf accounted for $60 \%$, about 100 people, more than 100, and increase to 2, 40000 people a year. Adverse drug reactions have become an important problem in harm to people's health [2].

\section{THE CURRENT STATUS OF OUR COUNTRY}

\section{A. The current status}

Through literature analysis to our country, summarizes the research status of adverse drug reactions and description, found that nearly 10 years, adverse drug reaction monitoring and research methods in common use in our country still is given priority to with case reports, case analysis is also more commonly used, but in most cases analysis for the current research and reviews research, literature review is becoming more and more be taken seriously. Clinical common adverse drug reaction monitoring and research method of case reports, case-control study, cohort study, adverse reaction monitoring, etc.

\section{B. The problems}

At present, our country uses the prospective study method in the study of adverse reactions. Survey, report to the relevant 
departments of personnel accounted for only $34 \%$ of adverse drug reactions. The provisions of the state, the content of the reports of adverse drug reactions and statistics is to strengthen the basis of drug regulatory and to guide rational drug use, not as a medical accident, medical lawsuits and processing on the basis of drug quality accident. But in the survey $86 \%$ of people think that adverse drug reactions related to medical disputes, this leads to the adverse drug reactions occurred when worry cause medical disputes report. As a result, reports of adverse drug reactions, especially new and severe adverse drug reactions report far short of the degree of "how many report how many", the report of good quality and value of the ratio of adverse drug reactions remains to be improved. In addition in the survey also found that most of the medical staff think adverse drug reactions occurred to the judgment standard, but for not understanding the concrete criterion, lead to the adverse drug reaction occurs can't make a right judgment, will be mistaken for a normal reaction of adverse drug reactions, a direct impact on the treatment of patients with even cause medical accidents.

In addition, because do not understand the reporting of adverse drug reactions, part of the medical staff in the case of adverse drug reactions report because I do not know how to report and give up, these are the report consciousness, lack of initiative in the adverse drug reaction monitoring work. From the survey found that the pharmacy staff's perception of adverse drug reactions in general knowledge or rules on integral cognition is superior to the doctors and nurses, but in the daily clinical activities but it has not been very good play to their own advantages, and other professional complementary [3].

\section{The previous reports}

Survey data show that most of the medical personnel system lack of understanding about the adverse drug reactions in their own units, individual leadership of adverse drug reaction monitoring work emphasis is insufficient, and failed to take reports of adverse drug reactions as medical institutions staff annual appraisal index, the medical staff could not regularly trained in the knowledge of adverse drug reactions, so that in the case of adverse drug reactions could not science to become an important reason for the high non-response rates of adverse drug reactions, and high level of hospital medical staff in the questionnaire overall cognitive water to plant a is much higher than that of low level of hospital, hospital information of adverse drug reaction monitoring work emphasis will directly affect the quality of the medical staff. Thus medical institutions at all levels should strengthen the degree of attention to the adverse drug reaction monitoring.

\section{REPORT SYSTEM, CLEAR RESPONSIBILITY AND LIABILITY}

Adverse drug reaction monitoring work in our country is still in its infancy, in around the city to carry out the adverse drug reaction monitoring work exists serious imbalance, monitoring low popularity, three has yet to establish a monitoring network in some areas, the basic unit personnel reported lack of motivation, high non-response rates, even some drug unit has no adverse drug reaction monitoring group, also is not specified personnel responsible for the work. Unit of each drug will work harder to further improve the quality and quantity of the reports of adverse drug reactions [4].

The world health organization in 1968 and the international drug monitoring plan set up the monitoring center. Early before the thalidomide incident, the United States on a single drug will set up a registration reporting system of adverse drug reactions, Britain, Japan and other developed countries and regions have established in the $1960 \mathrm{~s}$ adverse drug reaction monitoring and reporting system. Our country adverse drug reaction monitoring work began in 1988 and 2001 of the "drug administration law of the People's Republic of China" article defined "the state shall practice a system of adverse drug reactions report", adverse drug reactions monitoring in China formally entered the orbit of regulation.

International drug monitoring cooperation center for adverse drug reaction is defined as: the drugs in the prevention, diagnosis, treatment and regulate physiological function under normal usage and dosage, the harmful and unexpected reactions. The U.S. food and drug administration of adverse drug reactions are defined as drugs after regardless of whether any experience related to the drug, including any side effects, damage, allergic reactions, expectations of pharmacological effects.

\section{MONITORING OF ADVERSE DRUG REACTIONS}

Our country "the measures for the administration of adverse drug reaction report and monitoring of adverse drug reactions are defined as qualified drugs under normal usage and dosage has nothing to do with the purpose of or unexpected harmful response. It does not include fake medicines and medicines of inferior quality caused by reaction, or accidentally or intentionally caused by drug overdose reaction or drug use. Drug-induced disease refers to the prevention, diagnosis, treatment or regulate physiological function in the process, the human body function related to the drug clinical symptoms caused by abnormal or tissue damage. Drug-induced diseases and the relationship between the adverse drug reactions, adverse drug reaction is the source of drug-induced disease, and drug-induced disease is the result of the adverse drug reactions.

According to the WHO (World Health Operation, WHO) definition of adverse drug reactions known adverse drug reactions does not include the adverse reactions caused by abnormal usage. Therefore, can be thought of drug-induced disease generally do not include symptoms caused by abnormal usage. The reasonable classification of adverse drug reactions can reveal the relationship between drugs, make people pay attention to a common cause of similar reaction, so as to adopt similar measures to treat or prevent, epidemiological studies also needs to classify adverse drug reactions. Common classification methods are the classification of etiology, pathology classification, the severity classification and according to causality classification, etc. According to the etiology, adverse drug reactions are classified into three types. In general pharmacology therapy dose effect increased as A result, can often predict, a dose of relevance. In the crowd incidence is high, but the mortality rate is low. The side effects 
of drugs include toxic reaction, secondary reaction, but after effect, no tolerance and the withdrawal reaction, etc.

Functional change refers to the drug caused by the change of the human body organ or tissue function, the changes are mostly temporary. It can quickly return to normal after the drug was stopped, no changes, such as resistance to choline medicine and ganglion block caused by weak ileus, bradycardia caused by reserpine, etc.

The physical changes, caused by adverse drug reactions cause physical changes to physical changes and disease itself has no obvious difference, no specificity, we cannot according to histopathological examination, differential diagnosis and mainly according to the judgement of adverse drug reactions.

\section{A. The classification of the causation}

(1) Clear adverse drug reactions: the drug reaction in time is reasonable, or body fluids or tissues have measured corresponding drug concentration, the reaction involving drugs known form of reaction, reaction disappeared after drug withdrawal, give medication in response.

(2) It is possible (Probable) adverse drug reactions: drug reaction in time is reasonable, after reaction with drugs known function, reaction disappeared after the drug was stopped, and the patient's clinical characteristics known reaction can't explain.

(3) Possible adverse drug reactions: reaction time of the medicine is reasonable, the reaction to the role of the drug known, but the original clinical situations and other treatments can also lead to this kind of reaction.

(4) Conditional adverse drug reactions: reaction time of the medicine is reasonable, but the reaction performance is different from the drug known role, but the original clinical disease can also lead to this kind of reaction.

(5) Suspected adverse drug reactions: do not meet above other reaction of 4 kinds of circumstances.

\section{B. The classification of adverse drug reactions.}

Level 1: slight progress sexual response, such as a slight headache.

Level 2: the heavier than sexual response, such as severe headache.

Level 3: may affect the chronic effect of life, or for a period of time intermittently affect daily life, such as bronchial asthma, epilepsy.

Level 4: long-term impact the chronic effect of daily life, but not fatal or shorten the life of the effect.

Level 5: can shorten life expectancy, but does not directly endanger the life (such as high blood pressure).

Level 6:1.2 years can be lethal, but it is not emergency.

Level 7: the effect of emergency response, can be in one year, such as serious arrhythmia, systemic acute allergic.
The necessity of the adverse drug reactions monitoring drug prevention is the cure, regulate physiological function, improve the health level of important weapon, is related to people's life and health of special goods, not only requires the quality qualified, effective and safe use, but also the most key indicators and safety evaluation is its occurrence frequency and severity of adverse reactions. Only on post-marketing drug adverse reaction monitoring, in order to get a lot of information, and realize the scientific evaluation, in a timely manner to prevent and reduce the occurrence of adverse drug reactions, to guide clinical rational drug use and ensure the public safety [5].

With the continuous improvement of people's life quality requirements, the cognition of drugs can cause the body damage degree increasing, drug safety more and more get people's attention. Adverse drug reaction monitoring industry is a career that is related to the safety of the whole society, involving thousands of healthy body, also is the important basis of post-marketing drug evaluation again. And ensure the public safety (food) drug administration department, adverse drug reaction monitoring professional institutions, pharmaceutical producing enterprises, pharmaceutical trading enterprises and medical institutions of collective responsibility.

Our country is a quarter of the country has the world's population, with the increasing of new drug varieties, usage rate, group in frequency and quantity of per capital rising, adverse drug reactions will also increasingly prominent and severe. Literature reported in 1995, more than 11000 kinds of Chinese and western medicine in China, many Chinese herbs have been used for thousands of years, in the past few attaches great importance to the adverse drug reactions of traditional Chinese medicine research, little habits that adverse reactions caused by common Chinese patent medicine. As members of the international drug monitoring cooperation organization, drug supervision and administration of our country is not only to the Chinese people. Drug safety responsibility, but also contribute to the people of the world of drug safety, traditional Chinese medicine is our national treasure, and after the entry, the safety evaluation of Chinese traditional medicine of traditional Chinese medicine can move towards the world, plays a vital role. Therefore, no matter from the aspects of safe drug use at home and abroad, or international medical competition, are fully display the necessity and urgency of the adverse drug reaction monitoring work.

Pharmaceutical epidemiology is an application of epidemiological principle and method of study drug in the vast crowd, use and effect of the emerging disciplines in the clinical pharmacology and epidemiology of mutual penetration and development form a cross discipline, is the pharmaceutical epidemiology knowledge of theory, method and applied to the study of the use of drugs with the effect, with a large sample size, not intrusive, observational and natural real, etc. In practice, PE drug use information for the society to provide a wide range of people, to provide information on drug analysis the advantage and disadvantage of the application in the crowd, for drug post-marketing surveillance, listed drugs to evaluation and decision reference for the clinical rational drug use. Drug epidemiology will indicate drug reaction report and monitoring as the basis of the evaluation of drug safety, drug regulatory departments and the industry is as soon as possible for the drug 
safety signal is the only way, so is the basic method of drug alert.

In 2004 by the ministry of health and state food and drug administration to issue legal documents in the form of the adverse drug reaction report and monitoring measures for the administration, he every report management system, all aspects of the specific executive main body and clear rules. In recent years, our country's drug epidemiology and AD ding monitoring work has some great development, but not many big drug epidemiology research project, and compared with some developed countries in the world there is still a big gap.

\section{CONCLUSIONS}

Therefore, especially in such a society, just have been analysis, since it is everyone's forces staggered together to form resultant force to promote the development of the society, so social is supposed to safeguard its people you have medicine to cure illness, rather than completely according to the purchasing power of people and have no money to heal people to give up its for even the most basic health right, of course, according to people's buying power to get this is one aspect of medical resources, the high purchasing power can enjoy costly medical services. Speaking of which, may be some people think that social medical resources is too limited, not universal. But the society with its maximum capacity, meet the most basic medical needs, the vulnerable groups here are reflected in the field of medical and health care to "fair priority, and also give consideration to efficiency".

\section{REFERENCES}

[1] A.V. Kabanov. POLY(2-OXAZOLINE) BASED NANO FOR MULATIONS WITH SUPERIOR SAFETY AND EFFICACY IN VIVO[A].

[2] Shunjie Yan, Shifang Luan, Jinghua Yin. A NON-LEACHING BACTERIA-RESPONSIVE ANTIBACTERIAL SURFACE BASED ON A UNIQUE HIERARCHICAL ARCHITECTURE[A].

[3] Qian Si. Fluoroquinolone Clinical Analysis of Adverse Drug Reactions[A]. Proceedings of 2015 4th International Conference on Social Sciences and Society(ICSSS 2015 V73)[C]. 2015

[4] Yin-sheng ZHANG,Wei-hong CHEN,Hao-min LI. A Case Report of Medication Knowledge Translation in China[A]. Proceedings of 2014 International Conference on Energy, Environment and Materials Engineering(EEME 2014)[C]. 2014

[5] Fang LI, Minghui YANG, Jing NIE, Yuqi FENG, Chaohui YE, Maili Liu. Nmr Study on the Conformation of Valsartan and Related Compounds[A]. 